\title{
First aid for a damaged proteome
}

\author{
New insights into the regulatory mechanisms of protein folding and turnover are informing the \\ development of chemical tools and small molecules to treat proteostasis disorders.
}

Proteins receive constant insults from environmental stress or disease, but the overall integrity of the proteome remains largely intact, owing to a system that maintains protein homeostasis. The concept of a proteostasis network was proposed by Balch, Morimoto, Dillin and Kelly (Science 319, 916-919, 2008) and integrated principles of chaperone-mediated protein folding and ubiquitin-mediated degradation with the characterization of signaling pathways that were responsive to protein stress, such as the heat shock response and the unfolded protein response (UPR). The purpose of this network is to regulate protein synthesis, trafficking, disaggregation and degradation processes to ensure proper protein folding and to eliminate misfolded proteins when folding repair mechanisms are unsuccessful. Proteostasis research is now an interdisciplinary field using structural, chemical and biological information to understand the capacity of this network to restore protein shape and function and to apply this knowledge to treat disorders such as neurodegenerative or metabolic diseases. In this themed issue, we offer a collection of pieces focusing on advances in chemical biology that are improving our understanding of the mechanisms of proteostasis and how small-molecule modulation of these processes can be applied for therapeutic purposes.

Under normal cellular conditions, chaperones drive the folding of proteins to energetically favorable states, which are stabilized by weak intramolecular interactions. However, post-translational modifications are increasingly being seen as important regulators of protein folding and stability through intrinsic and extrinsic effects. Hebert and Kelly (Review, p. 902) point out that, in some cases, $\mathrm{N}$-linked glycosylated secretory proteins exhibit favorable folding energetics and low aggregation propensity through the formation of hydrogen bonds, hydrophobic contacts and $\mathrm{CH}-\pi$ interactions between glycans and protein. In addition, N-glycans are recognized by chaperones such as calnexin and calreticulin to enhance protein folding.

Despite effective natural mechanisms to ensure proper protein folding and trafficking, partially folded intermediates expose hydrophobic surfaces that have the potential to aggregate into toxic oligomers or fibrils. Gershenson, Gierasch, Pastore and Radford (Review, p. 884) propose that as proteins explore conformational landscapes for new functions, they also are at increased risk for revealing motifs that lead to aggregation. For example, the Josephin domain of the deubiquitinating enzyme Atx3 is an important motif for ubiquitin recognition but reveals an exposed hydrophobic surface that is also a hot spot for aggregation via polyglutamine tract expansion associated with neurodegenerative diseases.

To avoid the potential damaging effects of protein misfolding, cells use several natural quality control mechanisms for regulating proteostasis. As one example, the UPR system, which is activated in response to the accumulation of misfolded secretory proteins in the endoplasmic reticulum (ER), lowers ER protein flux by promoting chaperone expression, mRNA turnover and the selective relocalization of mRNA and ribosomes to the cytoplasm (Research Highlights, p. 877). Molinari (News and Views, p. 881) describes another protein clearance pathway, called rapid ER stress-induced export (RESET), which is active during the early stages of ER stress and results in the transport and degradation of misfolded GPI-anchored proteins. Under high or unresolvable ER stress, cells enact a terminal UPR program that results in apoptosis. Crews (Article, p. 957; News and Views, p. 879) makes use of a chemical biology tool that induces transient but resolvable ER stress to demonstrate a role for estrogen signaling at the later stages of this adaptive UPR pathway. Undoubtedly, future investigations will identify unique proteostatic pathways for particular types of proteins.

In addition to providing insights into proteostatic mechanisms, chemical biologists are at the forefront of efforts to identify small molecules that can reactivate the system. Morimoto (Elements, p. 875) and Lindquist (Review, p. 911) describe the important role that model organisms such as Caenorhabditis elegans and Drosophila have in identifying conserved proteostatic pathways with the potential to serve as targets of small-molecule compounds that could be developed for therapeutics for treating neurodegenerative diseases.
Efforts in targeting proteostatic networks with small molecules have already shown some promise. Recently, a small-molecule inhibitor of USP14, a deubiquitinating enzyme, was shown to enhance degradation of tau and reduce cellular toxicity (Nature 467, 179-184, 2010), suggesting that manipulation of the ubiquitin-proteasome system could be an attractive method to promote toxic protein clearance.

Alternatively, targeting kinase sensors for the UPR (IRE1 $\alpha$ and PERK) may offer another effective alternative for small-molecule regulation of proteostatic pathways, as outlined by Papa and Maly (Review, p. 892). Targeting related pathways may also have therapeutic benefits, as compounds that increase protein turnover by autophagy pathways can rescue mouse and neuronal models of TDP-43 aggregation (Nat. Chem. Biol. 10, 677-685, 2014).

Independent of the biological target, optimizing the specificity of chemical probes or drug leads remains an ongoing priority. King and Finley (Commentary, p. 870) provide some guidelines and principles to foster the development of more potent and specific activators and inhibitors of the ubiquitin enzymes E1, E2 and E3 and deubiquitinating enzymes. For example, disruption of multiple protein-protein interactions between E3 enzyme complexes and their cellular substrates may be required to ensure complete inactivation of this enzyme complex (Research Highlights, p. 876).

Despite the great progress in understanding the components and mechanisms of proteostasis, there remain several open questions that will require the development of biological and chemical tools as well as new cross-disciplinary perspectives. Morimoto (Elements, p. 875) challenges researchers to develop a global view of proteostasis in order to gain a better understanding of the complex interplay between molecules, cells and tissues. The ability to gain new mechanistic insight into both protein folding and stress responses, in combination with chemical probe approaches to alter the proteostasis network, are all areas where chemical biologists can contribute. We eagerly await the new insights that chemical biologists bring into the fold. 